\title{
Evolutionary Design of Magneto Rheological Brake for Two Wheeler
}

\author{
Prof. Shinde Vaibhav Vithal ${ }^{1}$, Dr.M.L.Kulkarni ${ }^{2}$ \\ ${ }^{1}$ (Mechanical Engineering, SVERI, Pandharpur, India) \\ ${ }^{2}$ (Mechanical Engineering, JSPM, Narhe, Pune, India)
}

\begin{abstract}
The proposed brake consists of multiple rotating discs immersed in Magneto Rheological Fluid and an enclosed electromagnet. When current is applied to electromagnet, Magneto Rheological Fluid solidifies as its yield stress varies as function of magnetic field. This controllable yield stress produces shear friction on rotating disc which generates braking torque. The braking torque value depends upon number of parameters like Current, Rotor Radius, Surface Area, Number of discs, Gap Size and Angular velocity. In order to minimize the stopping distance to $30 \mathrm{~cm}$ at response time of $0.5 \mathrm{sec}$, the brakes need to generate maximum torque up to level of $321 \mathrm{~N}-\mathrm{m}$ to have maximum braking effect. It is required to optimize the values of above parameters to get minimized stopping distance. Three configurations of Magneto Rheological brake are created and then for each configuration surface area is investigated then its effect on yield stress produced is investigated and then finally braking torque is calculated. Performance in terms of braking torque is evaluated then configuration which producing maximum torque is selected. It is found that brake having ring configuration shows maximum braking effect also it has advantage of compact size which is best suited for two wheeler application. Ring configuration Magneto Rheological Brake shows $10 \mathrm{~cm}$ stopping distance at 0.23 stopping time which is far better than existing drum type brake.
\end{abstract}

Keywords: Magneto Rheological Fluid brake, braking torque, stopping distance, two wheeler, Yield stress

Nomenclature: Ro outer radius, Ri Inner radius, tyd yield stress, $\mu$ p plastic viscosity, $\omega$ s rotational speed of disks, $h$ is gap thickness, $N$ is number of disk,Rlis cylinder radius, $R 2$ is ring inner radius, $R 3$ is ring outer radius, $B$ Magnetic field.

\section{Introduction}

The yield stress of Magneto Rheological Fluid can be controlled by varying the applied current [1]. The torque characteristics of Magneto Rheological Brake are controlled by regulating yield stress of Magneto Rheological Fluid [2]. On application of Magnetic field, Magneto Rheological Fluid changes its state from liquid to semi-solid. Ferromagnetic Particle aligns themselves in strong chain, due to such a chaining yield strength of fluid increases which opposes motion between stator and rotor and hence fulfills braking function [3]. A dynamic yield stress is function of the magnetic field and exponentially increases with respect to magnetic flux density [4].The torque transmissibility of an Magneto Rheological Brake depends much on properties of Magneto Rheological Fluid such as dynamic yield stress and viscosity [5].

To model behavior of Magneto Rheological Fluids the Bingham plastic model is used [6]. Magneto Rheological Braking torque depends upon material used, effective working surface, Magneto Rheological Fluid, applied current density and viscous torque of fluid [7]. The design and experimental evaluation of Magneto Rheological Brake has been done by $\mathrm{Li}$ and $\mathrm{Du}$ [8]. A dynamic finite element analysis was performed to study effect of applying repeated cycles of pressing and releasing brake on heat build up and showed that operating temperature can be intermittently reach outside Temperature range of fluid [9]. The limitations of gap plays a significant role in shear rate. In many researches, the particle gaps are typically 0.25 to $2 \mathrm{~mm}$ for Ease of manufacturing and assembly [10].Since aluminum ring is magnetically non-conductive it prevents magnetic circuit shorting around coil and forces it to go though Magneto Rheological Fluid gap [11]. In order to reduce weight and size of Magneto Rheological Brake the preferred ratio of Ri /Ro lies between 0.7 To 0.99 [12]. The performance of Magneto Rheological devices is relatively insensitive to temperature over a broad temperature range [13].Magneto Rheological Fluid is smart material whose rheological (viscosity) properties can be changed by Appling magnetic field. The yield strength can be controlled by current in the coil. This can be stated by Bingham Model as

$$
\tau=\tau \mathbf{H}+\mu \mathbf{p} \gamma
$$

where $\tau \mathrm{H}$ is the yield stress due to the applied magnetic field $\mathrm{H}, \mu \mathrm{p}$ is the constant plastic viscosity, which is considered equal to the no-field viscosity of the fluid, and $\gamma$ is the shear strain rate. 


\section{Methodology}

Three models of different configurations for disc are developed and there areas are calculated and then yield stress produced by each configuration is investigated. All the three cases are discussed here and finally results are compared with conventional drum brake to check the performance and evaluate optimized design.

\section{Configurations under Considerations}

Here different configurations are considered from perspective of their importance and usefulness. For simplicity in calculations of governing equations involved for Braking torque as given in following three cases some values are assumed. As per convenience constant values used in all Configurations are as shown in table.

Table No.1 Constant Values used for three Design Configurations

\begin{tabular}{|l|l|l|l|l|l|l|l|}
\hline $\mathbf{R o}(\mathbf{m})$ & $\mathbf{R i}(\mathbf{m})$ & $\mathbf{t}(\mathbf{m})$ & $\tau \mathbf{y d}(\mathbf{P a})$ & $\mu($ Pas-s) & $\omega(\mathbf{R a d} / \mathbf{s})$ & $\mathbf{L}(\mathbf{m})$ & $\mathbf{h}(\mathbf{m})$ \\
\hline 0.1 & 0.025 & 0.05 & 10000 & 0.214 & 44.7 & 0.05 & 0.01 \\
\hline
\end{tabular}

\subsection{Case1: Plane Disk}

In this case, it is assumed that a disk is mounted on shaft which is fitted on the bearings. Which are fixed with wheel shaft as shown in Fig. No.1The dimensions of disc Ro and Ri are given in table no.1for case I. Numbers of disk mounted on shaft are varied up to five and total torques obtained is calculated. The equation for Braking torque is created by integrating equation no.1 over entire disc surface. This configuration is selected because it is simple in design and disk can be mounted on shaft with press fit. It gives flexibility to increase number of disk to increase the torque. Equation no. 1 can be modified by integrating yield stress over entire surface area of disk having inner radius $\mathrm{Ri}$ and Outer radius Ro.

$$
\mathbf{T}=2 * 3.14 / 3 * \tau y^{*} h^{*}\left(\mathbf{R o}^{3}-\mathbf{R} i^{3}\right)+3.14 / 2 * h^{*} N * \mu P\left(\mathbf{R o}^{4}-\mathbf{R} i^{4}\right) * \omega s
$$

a)

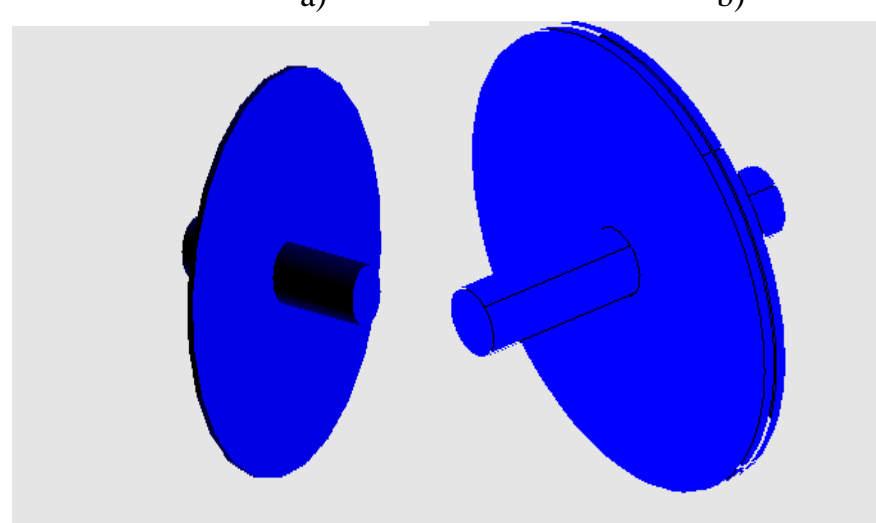

d)

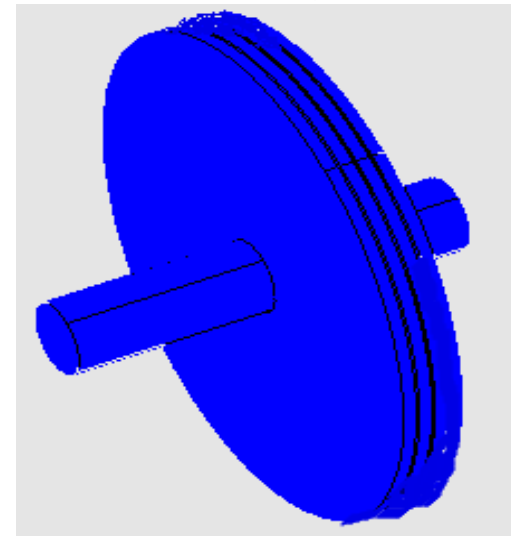

b)

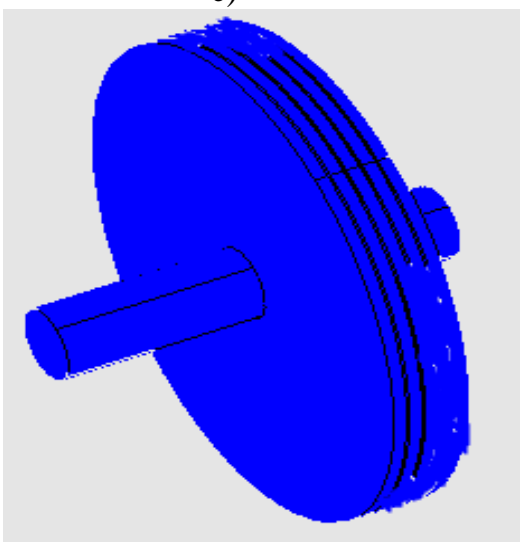

c)

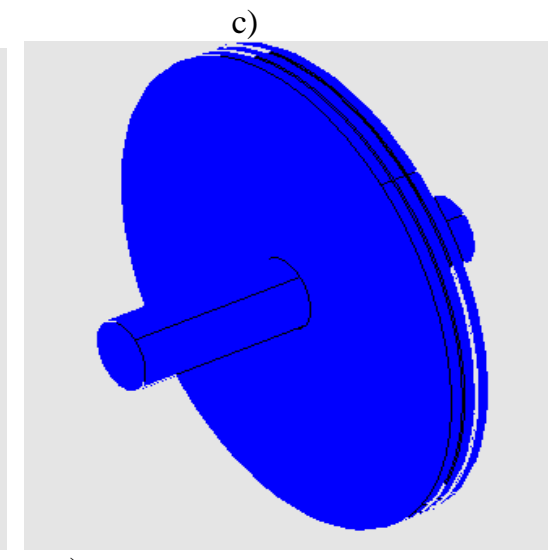

e)

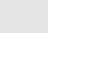




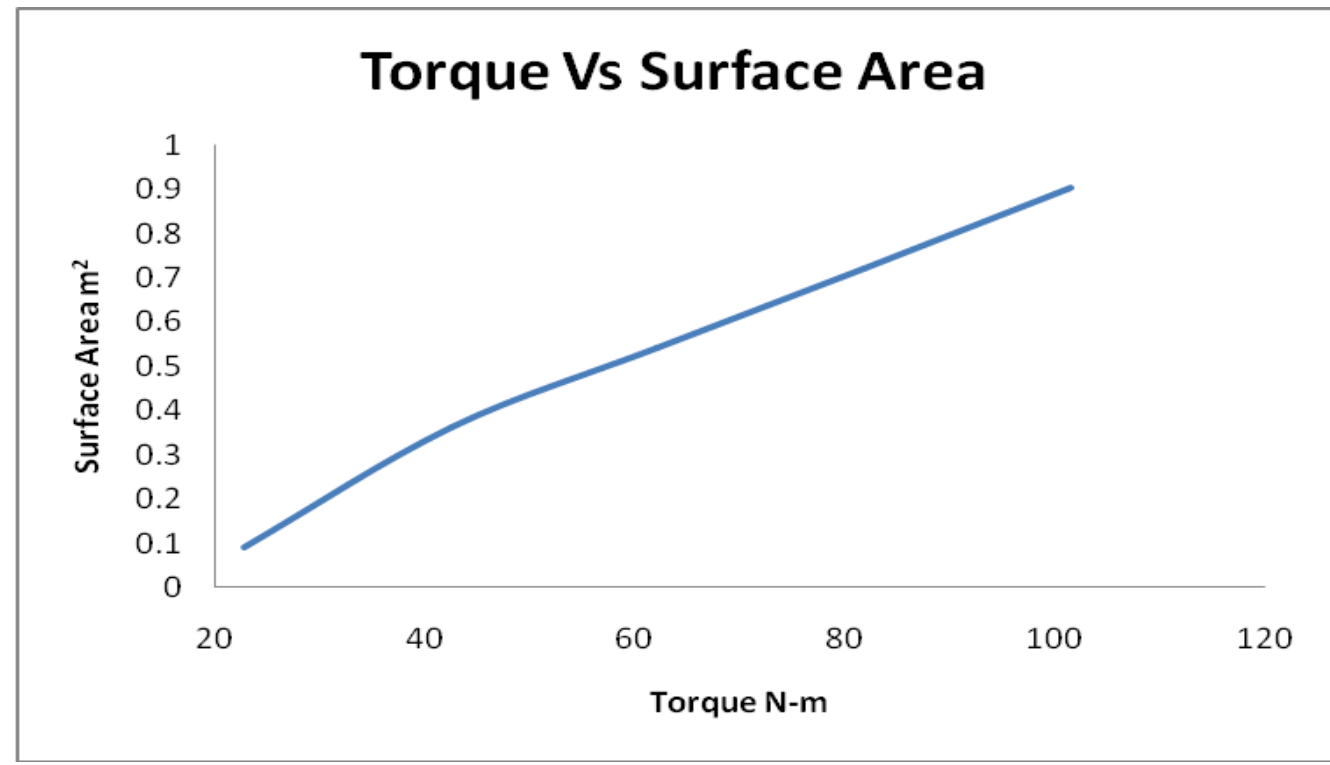

Fig. No.2 Braking Torque and Surface area for Configuration 1

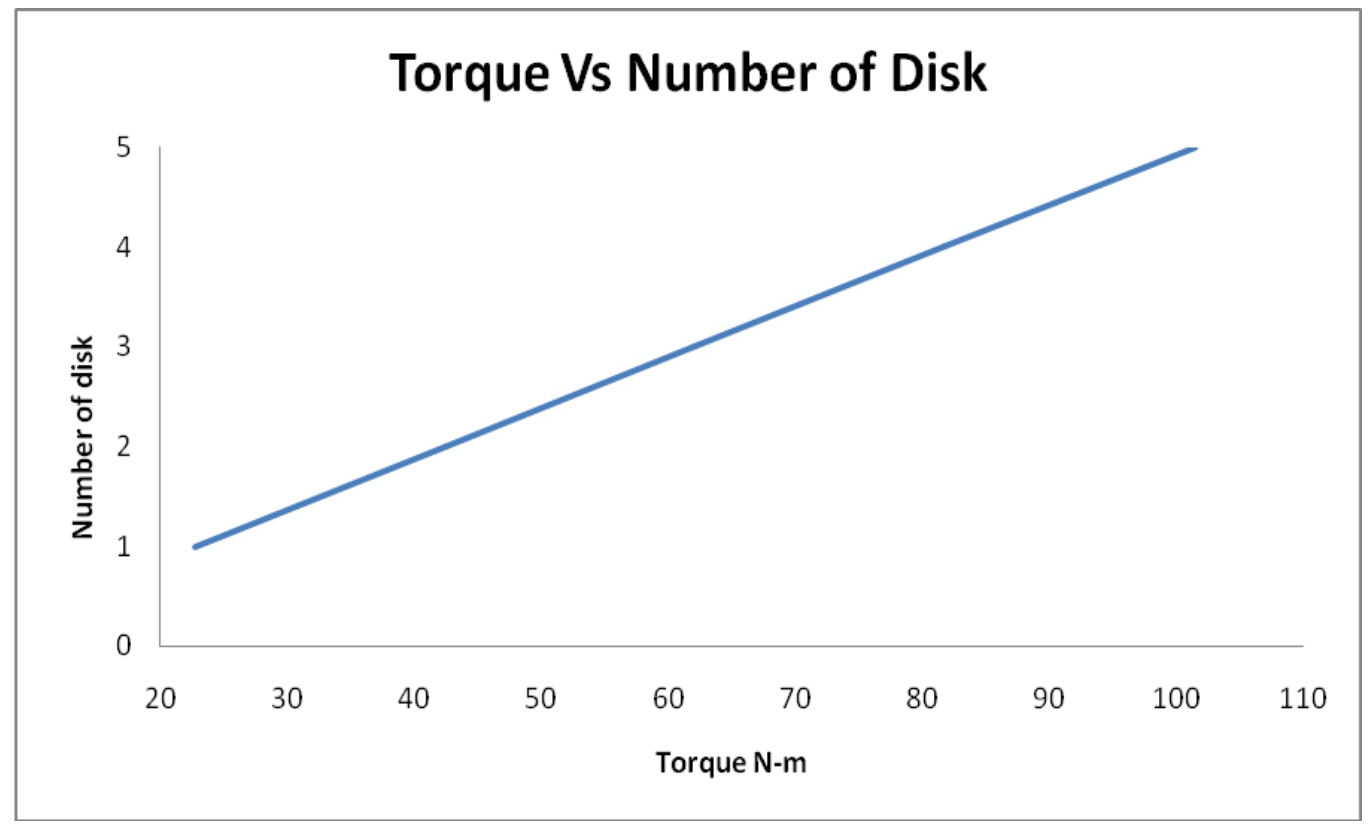

Fig No.3 Braking Torque and Number of Disk

It is found that on increasing number of disks mounted on shaft total braking torque of Magneto Rheological Brake increases linearly

\subsection{Case 2: Disk with Ring}

In this case, it is assumed that a disk with ring as shown in figure no. 4 is mounted on shaft which is fitted on the bearings which are fixed with wheel shaft. This configuration is selected because it is compact in size and ease in manufacturing due to simple in design and manufacturing.

Further Equation no.2 can be modified for Ring having inner radius R2 and Outer radius R3. These variables can be varied like R2 from 0.075 to 0.095 and R3 from 0.065 to 0.03 as shown in Table No.2 Then Braking torque values are calculated by using equation No.3.

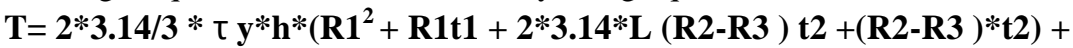
$4 / 3 * 3.14 * \eta^{*} w^{*} \mathbf{R} 1^{3}+4 / 3 * 3.14 * \eta * w^{*}\left(\mathbf{R 2}^{3}-\mathbf{R 3}^{3}\right)$ 
a)

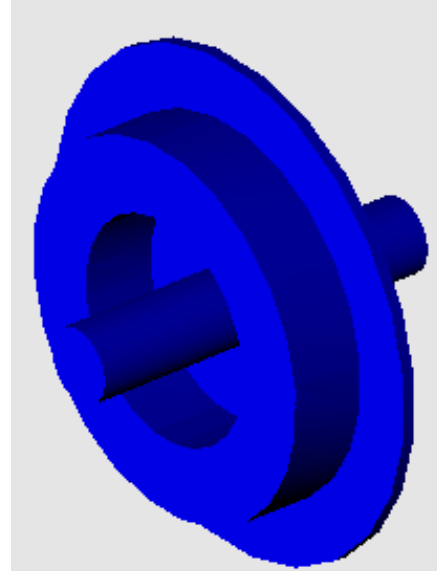

b)

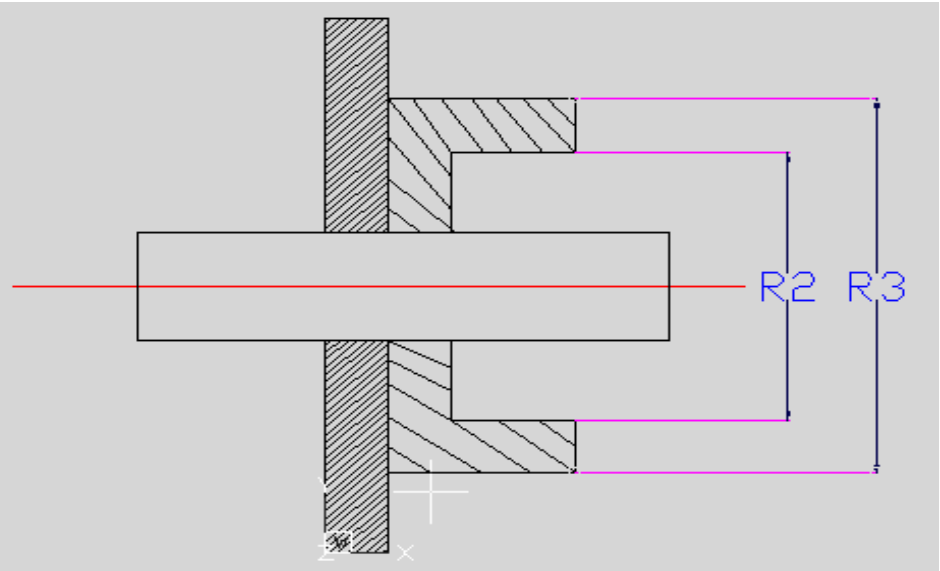

Figure No.4 a) Ring Mounted on Plane Disk b) Cross section shows Parameters R2 and R3 Used in Configuration II

Table No.2 Analytical Result for Ring with Inner Radius R2 and Outer Radius R3

\begin{tabular}{|l|l|l|l|l|l|l|l|l|l|l|}
\hline R2 $(\mathbf{m})$ & 0.075 & 0.085 & 0.095 & 0.075 & 0.085 & 0.095 & 0.095 & 0.095 & 0.095 & 0.095 \\
\hline R3 $(\mathbf{m})$ & 0.065 & 0.065 & 0.065 & 0.055 & 0.055 & 0.055 & 0.055 & 0.045 & 0.035 & 0.03 \\
\hline Surface Area $\left(\boldsymbol{m}^{2}\right)$ & 0.266 & 0.267 & 0.268 & 0.267 & 0.268 & 0.269 & 0.269 & 0.270 & 0.271 & 0.2715 \\
\hline Torque (N-m) & 16.72 & 16.78 & 16.85 & 16.78 & 16.85 & 16.913 & 16.91 & 16.97 & 17.04 & 17.07 \\
\hline
\end{tabular}

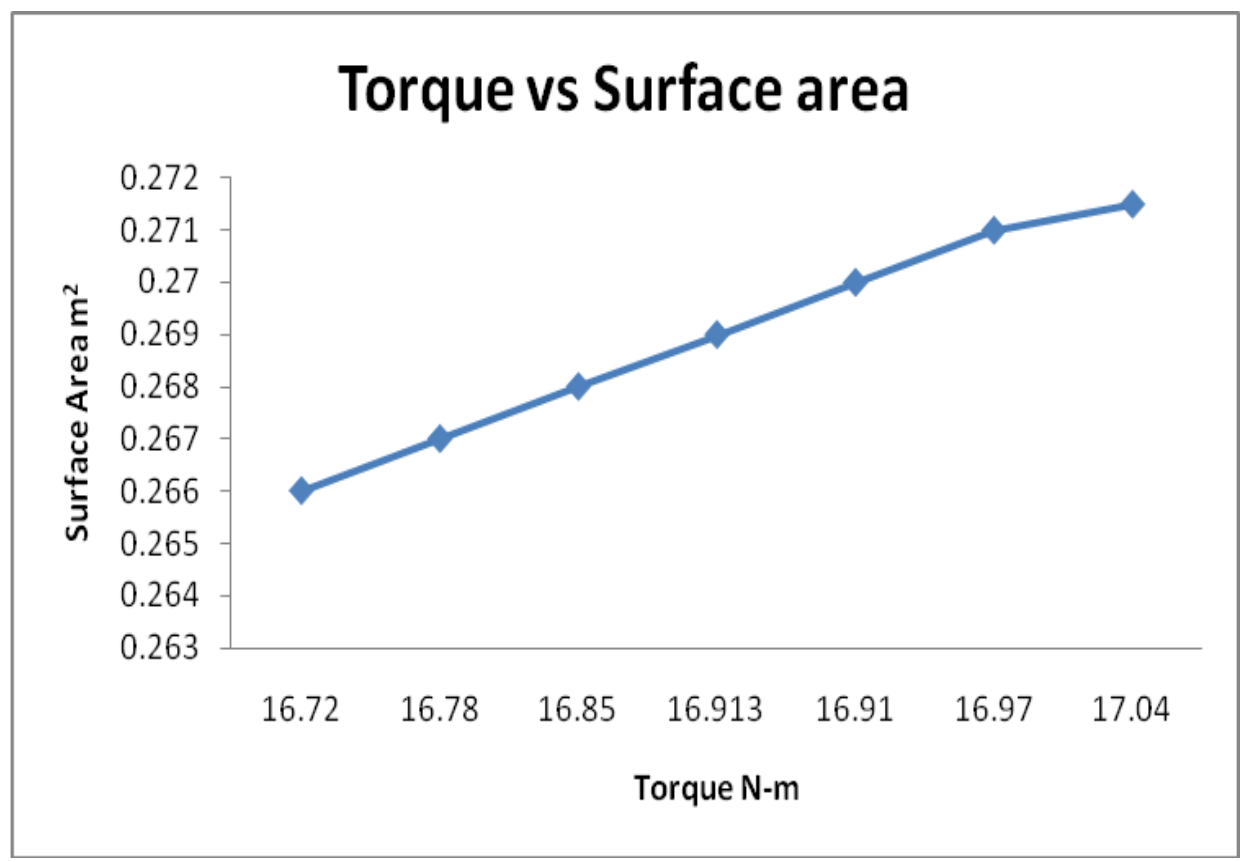

Fig. No.5 Braking Torque and Surface area for Configuration II

It is found that on increasing difference between R2 and R3 total surface area increases therefore braking torque of Magneto Rheological Brake increases linearly.

\subsection{Case 3: Disk with cylinders}

In this case it is assumed that a disk with cylinders on circumference as shown in fig. 3 is mounted on shaft which is fitted on the bearings which are fixed with wheel shaft.

Again equation No.2 can be modified for number of cylinders having length of cylinder L and Radius of cylinder as R1.Thse cylinders are mounted on shaft and their quantity is varied from 2 to 12 as shown in table no.3.Then braking torque values are calculated by using equation No.4.

$$
\begin{aligned}
& \mathrm{T}=2 * 3.14 * \tau \mathbf{y}^{*} \mathbf{h} *\left(\mathbf{R} 1^{2}+\mathbf{R} 1 \mathrm{t}+8 / 3 * 3.14 * \mathbf{R} 2^{2}+4 * 3.14 * \mathbf{R} 2 * \mathrm{~L}\right) \\
& +4 / 3 * 3.14 * \eta * \mathbf{R} 1^{3}+4 / 3 * 3.14 * \eta * w \mathbf{R} 2^{3}
\end{aligned}
$$


a)

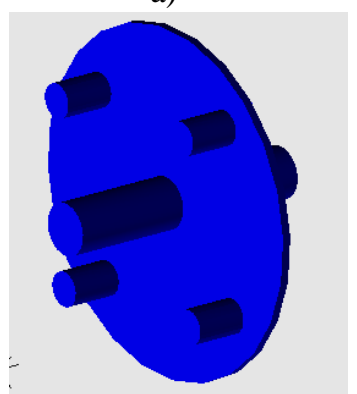

b)

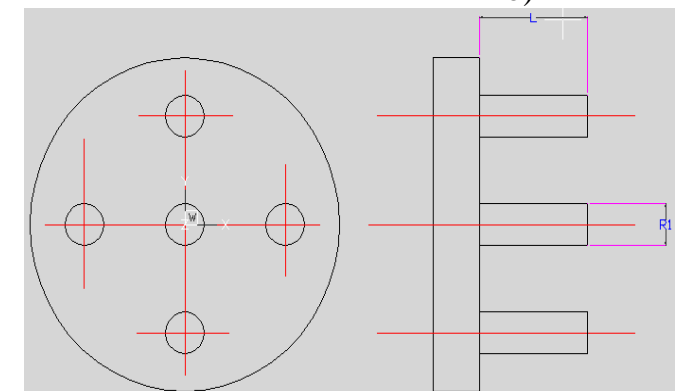

Figure No.6 a) Plane disk with four cylinders b) Side view shows parameters R1 and L used in Configuration III

Table No.3 Analytical Result for Case 3

\begin{tabular}{|l|l|l|l|l|l|l|l|}
\hline $\mathbf{2}$ * $\mathbf{L}$ R2 & 0.002 & 0.004 & 0.006 & 0.008 & 0.01 & 0.012 & 0.014 \\
\hline Surface area $\left(\mathbf{m}^{\mathbf{2}}\right)$ & 0.017 & 0.019 & 0.021 & 0.023 & 0.025 & 0.027 & 0.029 \\
\hline Torque & 12.87 & 14.38 & 15.90 & 17.41 & 18.92 & 20.44 & 21.95 \\
\hline
\end{tabular}

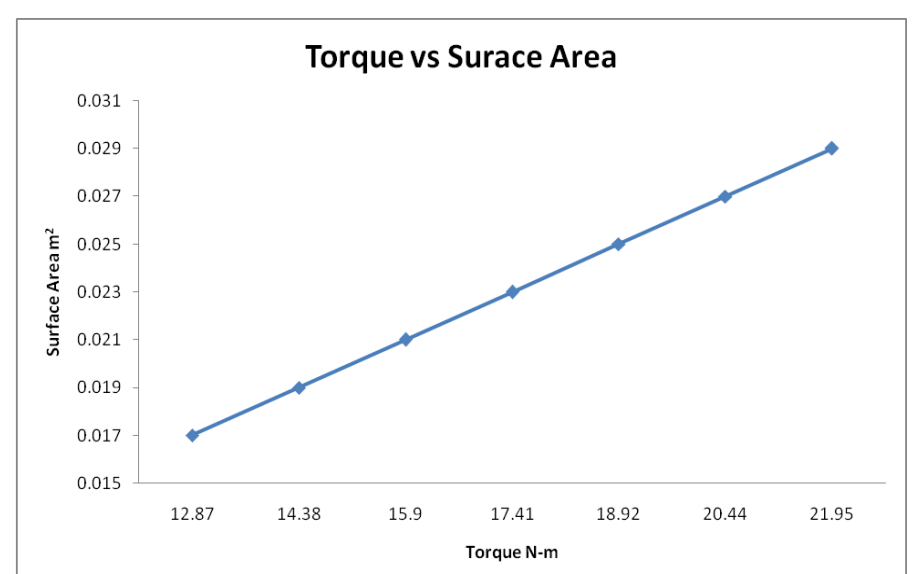

Fig. No. 7 Braking Torque and Surface Area for Configuration III

It is found that on increasing number of cylinders mounted on disk total braking torque of Magneto Rheological Brake increases linearly. As it is easier to increase number of cylinders mounted on disk this design is suitable due to its flexibility and simplicity.

\section{Results and Discussion}

For comparison purpose results obtained for Braking Torque for all three Configurations for different surface areas are as shown in Fig.No.6 It is clear that with increase in surface area braking torque increases. So within three configuration, Configuration No.2 i.e. disk with ring gives maximum surface area $0.26 \mathrm{~m}^{2}$ and therefore yield maximum torque up to $20 \mathrm{~N}-\mathrm{m}$.

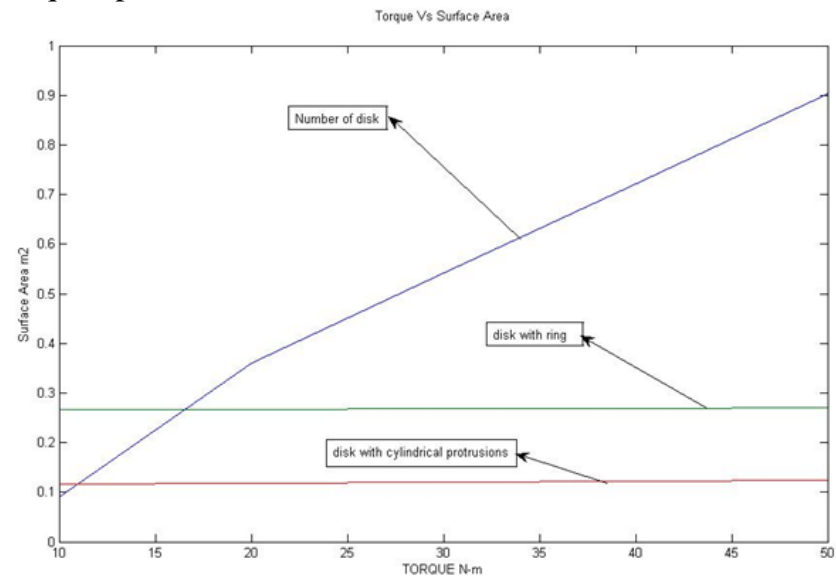

Fig. No.8 Comparison of Three Configurations 


\section{Experimental Results of Drum Brake}

An experiment is conducted on $125 \mathrm{cc}$ Glamour Hero Motor Bike by running the vehicle at $30 \mathrm{~km} / \mathrm{hr}$. and then brakes are applied. Braking time and Stopping distance for drum type brake is Measured by stopwatch and scale respectively. Results obtained are as shown in following table No.4

Table No.4 Braking time and Stopping Distance for Drum Brake

\begin{tabular}{|c|c|c|c|c|c|}
\hline Actuating pressure & Braking Time(s) & Stopping Time(s) & $\begin{array}{l}\text { Stopping } \\
\text { Distance(cm) }\end{array}$ & Engine Type & Torque \\
\hline $2 \mathrm{bar}$ & 0.5 & 0.5 & 62 & $109 \mathrm{cc}$ & $8.83 @ 5500 \mathrm{rpm}$ \\
\hline 3 bar & 0.5 & 0.52 & 120 & $125 \mathrm{cc}$ & 9BHP@7000pm \\
\hline 4 bar & 0.5 & 0.6 & 180 & $125 \mathrm{cc}$ & 9BHP@7000pm \\
\hline 5 bar & 0.5 & 0.63 & 190 & $125 \mathrm{cc}$ & 9BHP@7000pm \\
\hline 6bar & 0.5 & 0.8 & 270 & $125 \mathrm{cc}$ & 9BHP@7000pm \\
\hline
\end{tabular}

Analytically Expected Braking Time and Stopping distance for Magneto Rheological Brake are calculated.

\section{Conclusion}

It is found that performance of Magneto Rheological Brake for $125 \mathrm{CC}$ vehicle is far better than drum type braking system of existing vehicle in turns of stopping time and stopping distance. so design configuration II is more suited than I and III because of compactness in size and Maximum torque.

\section{Acknowledgements}

An Author acknowledge SVERI college of Engineering Pandharpur For giving help and support during this research Work.

\section{References}

[1] M.Keiu , R.Turezin, Properties and Application of Magneto Rheological Fluid, Journal of materials and manufacturing engineering , 18(1-2), (2006)

[2] Sarkar and Hirani, Design of a sqeeze film Magneto Rheological Brake considering compression enhanced shear yield stress of Magneto Rheological Fluid,13 ${ }^{\text {th }}$ International conference on Electro Rheological Fluid and Magneto Rheological suspensions, Journal of Physics , page 4-12, 2013

[3] S.Genc, Synthesis and properties of Magneto Rheological Fluids ,University of Pittsburg,2002 (PhD Thesis)

[4] J.M.Ginder, L.C.Davis, Shear stresses in Magneto Rheological Fluids: Role of Magnetic saturation, Applied physical letters, 34103412, 1994.

[5] J.D.Carlson,D.F.lerry et al , Controllable Brake, Us patent 5,Page no.842and 547,1998.

[6] Karakoc et al, Design considerations for automotive Magneto Rheological Brake, Elsevier, Mechatronics18, 434-447, (2008)

[7] W.H.Li and H. Du, Design and Experimental Evaluation of Magneto Rheological Brake ,International Journal of Advance Manufacturing Technology,508-515,2003

[8] Park EJ, Facao da Luz, A Multidisciplinary design optimization of Automotive Magneto Rheological Brake, Design comput Struc., 86(3-5):207-16, 2008

[9] Jung-BaeJun, Seong-Yong Uhan, Synthesis and Characterisation of mono disperse magnetic composite particles for Magneto Rheological Fluid Material, collides and surface A:-Physiochem.Engg. Aspects260(2005)157-164.

[10] Senkal D and Gurucak H, Spherical Brake with Magneto Rheological Fluid as Multi degree of freedom actuator for haptics, Journal of Mater Syst Struct ,20:2149-60,2009, Wasington DC,USA

[11] J.D.Carlson ,D.F.Leroy etal, Contollable Brake, Us patent( 5:542-47),1998.

[12] .V.V.shinde V.V. and M.L Kulkarni, Cylindrical Magneto Rheological Brake Design for two wheeler, Journal of Automobile Engineering and Application, V olume1 Issue 3,2015,India.

[13] Ahmad and Mohd, Experimental evaluation on Braking response of Magneto Rheological Brake",International journalof Metallurgy and engineering, Volume 1,issue3(2013) 